\title{
Correlated sampling in quantum Monte Carlo: a route to forces
}

\author{
Claudia Filippi \\ Department of Physics, National University of Ireland, Cork, Ireland \\ C. J. Umrigar \\ Cornell Theory Center and Laboratory of Atomic and Solid State Physics, Cornell University, Ithaca, New York 14853
}

(September 11, 2018)

\begin{abstract}
In order to find the equilibrium geometries of molecules and solids and to perform ab initio molecular dynamics, it is necessary to calculate the forces on the nuclei. We present a correlated sampling method to efficiently calculate numerical forces and potential energy surfaces in diffusion Monte Carlo. It employs a novel coordinate transformation, earlier used in variational Monte Carlo, to greatly reduce the statistical error. Results are presented for first-row diatomic molecules.

PACS numbers: 71.15.-m, 31.10.+z, 31.25.Nj, 02.70.Lq
\end{abstract}

Over the past decade, quantum Monte Carlo (QMC) methods [1] have been used to calculate the structural and electronic properties of a variety of atoms, clusters and solids. For systems with large numbers of electrons, QMC methods at present provide the most accurate benchmark calculations of structural energies. However, a major difficulty of QMC methods has been the determination of equilibrium geometries and potential energy surfaces. Hence, most QMC calculations have been performed on geometries obtained with either density functional theory (DFT) or conventional quantum chemistry methods. The computation of forces on nuclei has been a stumbling block that has limited a more widespread use of QMC methods.

DFT methods or standard quantum chemistry techniques use the Hellman-Feynman theorem to compute the forces on nuclei 断. Unfortunately, this is not practical within QMC for three reasons. First, the wave functions used in QMC calculations are usually not obtained by minimizing the energy. Therefore, if HellmanFeynman theorem were employed in variational Monte Carlo (VMC), the forces would have a systematic error. Second, in fixed-node diffusion Monte Carlo (DMC) the Hellman-Feynman force has an error due to the discontinuity in the derivative of the fixed-node wave function at nodes [5]. Finally, in both VMC and DMC, the statistical fluctuations would be too large since the fluctuations of the potential energy are much larger than those of the total energy.

Alternatively, one could simply compute energy differences to obtain either forces (for an infinitesimal displacement of the ions) or the full potential energy surface of the system. However, while quantum chemistry methods can rely on having an approximately constant and smoothly varying error in the energy, a major disadvantage of QMC methods is that, in addition to systematic errors, one has statistical errors which make the determination of energy differences or smooth potential energy surfaces very expensive in computer time. Even though it is not possible to entirely eliminate the statistical errors, it is possible, by using correlated sampling [6], to make the statistical errors in the relative energies of different geometries much smaller than the errors in the separate energies and to make them vanish in the limit that the two geometries become identical. In the past, the correlated sampling technique has been used within VMC [7]:8] but there have been very few attempts 9 to extend the approach to DMC, and these were approximate and/or inefficient and were tested only on $\mathrm{H}_{2}, \mathrm{H}_{3}^{+}$and $\mathrm{LiH}$.

In this paper, we present a novel DMC correlated sampling technique to efficiently compute accurate forces and potential energy surfaces. The DMC bond lengths of first-row diatomic molecules computed with this algorithm are found to be in better agreement with experiment values than are the VMC, Hartree-Fock (HF), local density approximation (LDA) and generalised gradient approximation (GGA) values.

Correlated sampling in variational Monte Carlo. Instead of performing independent VMC runs which would have independent statistical errors, one generates $\mathrm{MC}$ configurations for a reference situation only. The MC configurations are sampled from $\psi^{2}$ where $\psi$ is the wave function for the reference situation. Then, unbiased expectation values for somewhat different secondary wave functions $\psi_{\mathrm{s}}$ are obtained by reweighting the configurations sampled from $\psi^{2}$, e.g., for Hamiltonians $\mathcal{H}$ and $\mathcal{H}_{\mathrm{s}}$,

$$
\begin{aligned}
E_{\mathrm{s}}-E & =\frac{\left\langle\psi_{\mathrm{s}}\left|\mathcal{H}_{\mathrm{s}}\right| \psi_{\mathrm{s}}\right\rangle}{\left\langle\psi_{\mathrm{s}} \mid \psi_{\mathrm{s}}\right\rangle}-\frac{\langle\psi|\mathcal{H}| \psi\rangle}{\langle\psi \mid \psi\rangle} \\
& =\frac{1}{N_{\text {conf }}} \sum_{i=1}^{N_{\mathrm{conf}}}\left\{\frac{\mathcal{H}_{\mathrm{s}} \psi_{\mathrm{s}}\left(\mathbf{R}_{i}\right)}{\psi_{\mathrm{s}}\left(\mathbf{R}_{i}\right)} W_{i}-\frac{\mathcal{H} \psi\left(\mathbf{R}_{i}\right)}{\psi\left(\mathbf{R}_{i}\right)}\right\},
\end{aligned}
$$

where the weights of the $N_{\text {conf }} \mathrm{MC}$ configurations are

$$
W_{i}=\frac{N_{\text {conf }}\left|\psi_{\mathbf{s}}\left(\mathbf{R}_{i}\right) / \psi\left(\mathbf{R}_{i}\right)\right|^{2}}{\sum_{i=1}^{N_{\text {conf }}}\left|\psi_{\mathbf{s}}\left(\mathbf{R}_{i}\right) / \psi\left(\mathbf{R}_{i}\right)\right|^{2}},
$$

and $\mathbf{R} \equiv\left(\mathbf{r}_{1}, \ldots, \mathbf{r}_{N}\right)$. The statistical error in $E_{\mathrm{s}}-E$ is considerably smaller than that in $E_{\mathrm{s}}$ or $E$, making this 
method of calculating $E_{\mathrm{s}}-E$ more efficient than performing independent VMC computations of $E_{\mathrm{s}}$ and $E$.

Space-warp coordinate transformation. Since we want to compute the relative energy of two different geometries, the Hamiltonians $\mathcal{H}$ and $\mathcal{H}_{\mathrm{s}}$ in Eq. 1 correspond to two sets of nuclear coordinates, $\mathbf{R}_{\alpha}$ and $\mathbf{R}_{\alpha}^{\mathrm{s}}$, for the reference and the secondary geometry, respectively.

The electronic coordinates sampled from the reference wave function $\psi^{2}$ will not be optimal for computing the energy $E_{\mathrm{s}}$ corresponding to the nuclear coordinates $\mathbf{R}_{\alpha}^{\mathrm{s}}$, since the electron density will be peaked at $\mathbf{R}_{\alpha}$ rather than at $\mathbf{R}_{\alpha}^{\mathrm{s}}$. To solve this problem, a mapping of the electron coordinates was introduced in Ref. [8] such that those electrons that are close to a given nucleus move almost rigidly with that nucleus:

$$
\mathbf{r}_{i}^{\mathrm{s}}=\mathbf{r}_{i}+\sum_{\alpha=1}^{N_{\text {atoms }}}\left(\mathbf{r}_{\alpha}^{\mathrm{s}}-\mathbf{r}_{\alpha}\right) \omega_{\alpha}\left(\mathbf{r}_{i}\right)
$$

where

$$
\omega_{\alpha}\left(\mathbf{r}_{i}\right)=\frac{F\left(\left|\mathbf{r}_{i}-\mathbf{r}_{\alpha}\right|\right)}{\sum_{\beta=1}^{N_{\text {atoms }}} F\left(\left|\mathbf{r}_{i}-\mathbf{r}_{\beta}\right|\right)} ; \quad \sum_{\alpha=1}^{N_{\text {atoms }}} \omega_{\alpha}\left(\mathbf{r}_{i}\right)=1 .
$$

(We use Latin indices for electronic coordinates and Greek indices for nuclear coordinates.) $F(r)$ is any sufficiently rapidly decaying function such as $r^{-\kappa}$, $\exp (-\kappa r)$ or $\exp (\kappa / r)$. The reduction in statistical error due to the use of these warped electronic coordinates for the secondary geometries is large and almost independent of the choice for $F(r)$ and $\kappa$. Unless otherwise stated, all results in this paper were computed with $F(r)=r^{-\kappa}$ and $\kappa=4$.

The equation for $E_{\mathrm{s}}-E$ (Eq. 国) is now

$$
E_{\mathrm{s}}-E=\frac{1}{N_{\text {conf }}} \sum_{i=1}^{N_{\text {conf }}}\left(\frac{\mathcal{H}_{\mathrm{s}} \psi_{\mathrm{s}}\left(\mathbf{R}_{i}^{\mathrm{s}}\right)}{\psi_{\mathrm{s}}\left(\mathbf{R}_{i}^{\mathrm{s}}\right)} W_{i}-\frac{\mathcal{H} \psi\left(\mathbf{R}_{i}\right)}{\psi\left(\mathbf{R}_{i}\right)}\right),
$$

where

$$
W_{i}=\frac{N_{\text {conf }}\left|\psi_{\mathbf{s}}\left(\mathbf{R}_{i}^{\mathrm{s}}\right) / \psi\left(\mathbf{R}_{i}\right)\right|^{2} J\left(\mathbf{R}_{i}\right)}{\sum_{j=1}^{N_{\text {conf }}}\left|\psi_{\mathbf{s}}\left(\mathbf{R}_{j}^{\mathrm{s}}\right) / \psi\left(\mathbf{R}_{j}\right)\right|^{2} J\left(\mathbf{R}_{j}\right)},
$$

and $J(\mathbf{R})$ is the Jacobian for the transformation (Eq. 3).

Correlated sampling in diffusion Monte Carlo. In DMC, the imaginary-time evolution operator $\exp (-\mathcal{H} \tau)$ is used to project out the ground state from the trial wave function within the fixed-node and the short-time approximations [10]. The primary walk is generated according to the stochastic implementation of the integral equation:

$$
f\left(\mathbf{R}^{\prime}, t+\tau\right)=\int d \mathbf{R} G\left(\mathbf{R}^{\prime}, \mathbf{R}, \tau\right) f(\mathbf{R}, t),
$$

where $G\left(\mathbf{R}^{\prime}, \mathbf{R}, \tau\right)=\left\langle\mathbf{R}^{\prime}|\exp \{-\mathcal{H} \tau\}| \mathbf{R}\right\rangle$. If we introduce importance sampling using the reference wave function $\psi$, the importance sampled Green's function for small values of $\tau$ (short-time approximation) is given by the product of three factors, diffusion, drift and growth/decay:

$$
\tilde{G}\left(\mathbf{R}^{\prime}, \mathbf{R}, \tau\right)=\frac{1}{(2 \pi \tau)^{\frac{3 N}{2}}} e^{-\frac{\left(\mathbf{R}^{\prime}-\mathbf{R}-\mathbf{V}(\mathbf{R}) \tau\right)^{2}}{2 \tau}} e^{S\left(\mathbf{R}^{\prime}, \mathbf{R}, \tau\right)},
$$

where $\mathbf{V}=\nabla \psi(\mathbf{R}) / \psi(\mathbf{R})$ and $S\left(\mathbf{R}^{\prime}, \mathbf{R}, \tau\right)=\left(E_{\mathrm{T}}-\right.$ $\left.E_{\mathrm{L}}\left(\mathbf{R}^{\prime}\right)-E_{\mathrm{L}}(\mathbf{R})\right) \tau / 2$ with $E_{\mathrm{L}}=\mathcal{H} \psi(\mathbf{R}) / \psi(\mathbf{R})$. At time $t$, a set of primary walkers characterized by the pairs $\left(\mathbf{R}_{i}, w_{i}\right)$ is a random realization of the distribution $f$ :

$$
f(\mathbf{R}, t)=\sum_{i} w_{i} \delta\left(\mathbf{R}-\mathbf{R}_{i}\right)
$$

Each walker executes a branching random walk: a walker originally at $\mathbf{R}$ drifts to $\mathbf{R}+\mathbf{V}(\mathbf{R}) \tau$ and then diffuses to $\mathbf{R}^{\prime}$ according to the Gaussian term in Eq. 8. To ensure that, in the limit of perfect importance sampling (i.e. $\psi$ is the ground state wave function $\psi_{0}$ ), we are sampling $\psi^{2}$ despite the short-time approximation in the Green's function, the move is accepted with probability

$$
p=\min \left\{1, \frac{\left|\psi\left(\mathbf{R}^{\prime}\right)\right|^{2} \tilde{T}\left(\mathbf{R}, \mathbf{R}^{\prime}, \tau\right)}{|\psi(\mathbf{R})|^{2} \tilde{T}\left(\mathbf{R}^{\prime}, \mathbf{R}, \tau\right)}\right\},
$$

as prescribed by the detailed balance condition. We denote by $\tilde{T}$ the drift-diffusion part of the Green's function $\tilde{G}$. Finally, the weight of the walker is multiplied by $\exp \left[S\left(\mathbf{R}^{\prime}, \mathbf{R}, \tau\right)\right]$. In practice, we employ the modified version of $\tilde{G}$ presented in Ref. [11] that takes into account the non-analyticities of $\mathbf{V}$ and $E_{\mathrm{L}}$ at the nodes and particle coalescence points.

Given a primary walk generated according to Eq. 8, the secondary walk is specified by the space-warp transformation (Eq. 3). Two complications, absent in VMC, arise for correlated sampling in DMC. First of all, the dynamics of the secondary walker should have been governed by an importance sampled Green's function constructed from the secondary wave function $\psi_{\mathrm{s}}, \tilde{G}_{\mathrm{s}}\left(\mathbf{R}^{\mathrm{s} \prime}, \mathbf{R}^{\mathrm{s}}, \tau\right)$, and the move should have been accepted with probability

$$
p_{\mathrm{s}}=\min \left\{1, \frac{\left|\psi_{\mathrm{s}}\left(\mathbf{R}^{\mathrm{s} \prime}\right)\right|^{2} \tilde{T}_{\mathrm{s}}\left(\mathbf{R}^{\mathrm{s}}, \mathbf{R}^{\mathrm{s} \prime}, \tau\right)}{\left|\psi_{\mathrm{s}}\left(\mathbf{R}^{\mathrm{s}}\right)\right|^{2} \tilde{T}_{\mathrm{s}}\left(\mathbf{R}^{\mathrm{s} \prime}, \mathbf{R}^{\mathrm{s}}, \tau\right)}\right\} .
$$

However, the secondary-geometry move was effectively proposed according to the drift-diffusion Green's function $\tilde{T}\left(\mathbf{R}^{\prime}, \mathbf{R}, \tau\right) / J\left(\mathbf{R}^{\prime}\right)$ and accepted with probabilty $p$ defined in Eq. 10. To correct for the wrong dynamics, we should multiply the weights of the secondary walkers by

$$
r \frac{\tilde{G}_{\mathrm{s}}\left(\mathbf{R}^{\mathrm{s}}, \mathbf{R}^{\mathrm{s}}, \tau\right)}{\tilde{T}\left(\mathbf{R}^{\prime}, \mathbf{R}, \tau\right) / J\left(\mathbf{R}^{\prime}\right)},
$$

where $r=p_{\mathrm{s}} / p$ if the move is accepted and $r=(1-$ $\left.p_{\mathrm{s}}\right) /(1-p)$ if the move is rejected. However, these products fluctuate wildly ( $r$ can be anywhere between zero 
and infinity). Therefore, it is not practical to follow this route to perform correlated sampling unless bounds can be placed on the ratios while at the same time ensuring that unbiased results are obtained in the $\tau \rightarrow 0$ limit.

An additional complication is the common practice in fixed-node DMC to reject moves that cross nodes. If a primary walker attempts to cross, the move is rejected, $p$ in Eq. 10 is set to zero, and the ratio $r$ for the secondary walker (Eq. 12) becomes ill-defined. Moreover, if primary and secondary walkers were to be treated on the same footing ( $p_{\mathrm{s}}$ set to zero when the secondary walker crosses its own nodes), the weights of the secondary walkers would all become zero in a sufficiently long run. Even though this problem can be easily overcome since it is legitimate to do fixed-node DMC allowing walkers to cross nodes [11], reweighting as in Eq. 12 remains impractical due to the large fluctuations.

In this paper, we propose an alternative correlated sampling algorithm. Our algorithm is approximate but very accurate. Given the successful implementation of correlated sampling within $\mathrm{VMC}$, we wish to devise a scheme that differs as little as possible from VMC but yields results very close to the fixed-node DMC limit for the secondary geometries. We perform the primary walk as described above and generate the secondary walks according to the space-warp transformation. In the averages, we retain the ratios of the secondary and primary wave functions as would be done in VMC (Eqs. 5 and (6). The secondary weights are the primary ones multiplied by the product of the factors $\exp \left[S_{\mathrm{s}}\left(\mathbf{R}^{\mathrm{s} \prime}, \mathbf{R}^{\mathrm{s}}, \tau_{s}\right)-S\left(\mathbf{R}^{\prime}, \mathbf{R}, \tau\right)\right]$ for the last $N_{\text {proj }}$ generations. Note that, in the exponential factors, we introduced $\tau_{\mathrm{s}}$, a time-step for the secondary path, in general different from the time-step $\tau$ used for the primary walk. Due to the warp transformation, the secondary moves were effectively proposed with a different time-step, $\tau_{s}$, in the drift-diffusion term. A sensible definition of $\tau_{\mathrm{s}}$ is

$$
\tau_{\mathrm{s}}=\tau \frac{\left\langle\Delta R_{\mathrm{s}}^{2}\right\rangle}{\left\langle\Delta R^{2}\right\rangle},
$$

where $\Delta R$ is the displacement resulting from diffusion, and $\Delta R_{\mathrm{s}}$ is the displacement needed to take the secondary walker from its drifted position to the position specified by the space-warp transformation. Having computed $\tau_{\mathrm{s}}$ over the first equilibration blocks of our DMC run, we will use this time-step for computing the drift and reweighting of the secondary walk. Therefore, each secondary geometry has a different time-step $\tau_{\mathrm{s}}$.

Secondary geometry wave functions. We considered three choices for secondary geometry wave functions:

(1) The secondary geometry wave functions have the same parameters $\{\mathbf{p}\}$ as the primary one but the coordinates are relative to the new nuclear positions: $\psi_{\mathrm{s}}\left(\mathbf{R}_{i}, \mathbf{R}_{\alpha}^{\mathrm{s}}\right)=\psi\left(\mathbf{R}_{i}, \mathbf{R}_{\alpha}^{\mathrm{s}}, \mathbf{p}_{\mathrm{s}}\right)$ with $\mathbf{p}_{\mathrm{s}}=\mathbf{p}$, possibly with the minimal changes required to impose the cusp conditions.
(2) The secondary geometry wave functions at warped electron positions are related to the primary ones at the original positions, $\psi_{\mathrm{s}}\left(\mathbf{R}_{i}^{\mathrm{s}}, \mathbf{R}_{\alpha}^{\mathrm{s}}\right)=\psi\left(\mathbf{R}_{i}, \mathbf{R}_{\alpha}, \mathbf{p}\right) / \sqrt{J\left(\mathbf{R}_{i}\right)}$. This wave function depends on the transformation (it was used in Ref. [9, $b-c$ ] with a different transformation) and has the advantage that the weights $W_{i}$ in (Eq. 2) are unity. Surprisingly, it gives larger fluctuations of the energy differences than choice (1).

(3) $\psi_{\mathrm{s}}\left(\mathbf{R}_{i}, \mathbf{R}_{\alpha}^{\mathrm{s}}\right)=\psi\left(\mathbf{R}_{i}, \mathbf{R}_{\alpha}^{\mathrm{s}}, \mathbf{p}_{\mathrm{s}}\right)$ with reoptimized parameters $\mathbf{p}_{\mathbf{s}}$. This choice gives the smallest fluctuation of the energy differences and the best potential energy surface. We calculate all molecules with choice (1) but also demonstrate the superiority of choice (3) for $\mathrm{B}_{2}$.

Results and conclusions. The algorithms presented in the previous sections are tested on first-row homonuclear dimers. The primary wave functions [3] were optimized close to the experimental bond length by the variance minimization method [2]. The potential energy curves were obtained with correlated sampling from ten geometries, using the warp transformation and recentered secondary geometry wavefunctions (choice (1) above).

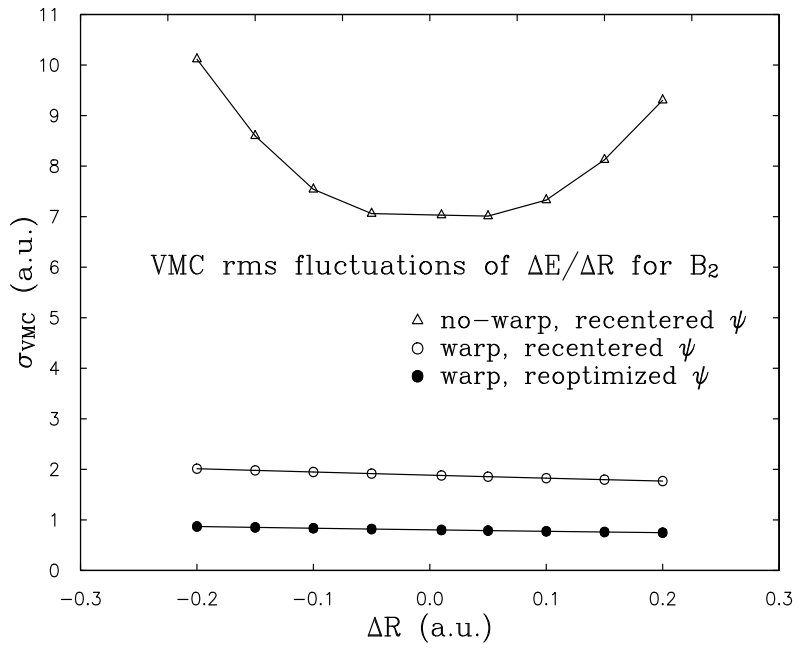

FIG. 1. VMC fluctuations $\left(\sigma_{\mathrm{VMC}}\right)$ of the relative energy of the primary and secondary geometries divided by the bond stretch for $\mathrm{B}_{2}$. The smallest $\sigma_{\mathrm{VMC}}$ is achieved by using warping along with reoptimized secondary wave functions.

To ascertain the efficiency of our method, we performed two additional calculations for $\mathrm{B}_{2}$; in the first, we omitted the warp transformation, whereas in the second we employed reoptimized, rather than recentered, secondary wavefunctions. In Fig. 1, we present the VMC root-mean-square fluctuations $\left(\sigma_{\mathrm{VMC}}\right)$ of the relative energy of primary and secondary geometries divided by the atomic displacement, $\Delta E / \Delta R$, for $\mathrm{B}_{2}$. Introducing the warp transformation yields a reduction of about a factor of 3.5-5 in $\sigma_{\mathrm{VMC}}$, which corresponds to a factor of $12-25$ saving in computer time. Moreover, $\sigma_{\mathrm{VMC}}$ is only slightly dependent on the secondary geometry used. As expected, a further reduction in $\sigma_{\mathrm{VMC}}$ is obtained when the spacewarp transformation is used in combination with reoptimized, rather than recentered, secondary geometry wave 
functions. The space-warp transformation was found to be of even greater help for heavier molecules, e.g. for $\mathrm{F}_{2}$ the reduction in the fluctuations was a factor of 3.2-8.2.

To test the accuracy of our DMC correlated sampling algorithm, we performed DMC runs for $\mathrm{H}_{2}$ and $\mathrm{B}_{2}$ for three different primary geometries, (a) the equilibrium geometry, (b) a geometry stretched by 0.2 and (c) by -0.2 a.u. The runs (a), (b) and (c) should give identical potential energy curves if the algorithm were exact. In Fig. 2, we show results for $\mathrm{B}_{2}$ that reveal the high accuracy of our DMC algorithm: the three DMC curves are very close and clearly distinguishable from the VMC results. These results are confirmed by the calculations for $\mathrm{H}_{2}$ where, despite the use of an intentionally poor wave function, the three curves gave the equilibrium bond lengths (a) 1.4014(2) (b) 1.4014(2) and (c) 1.4015(2) a.u. The true equilibrium bond length, from a careful fit to the results of Ref. [12], is 1.4011 a.u.

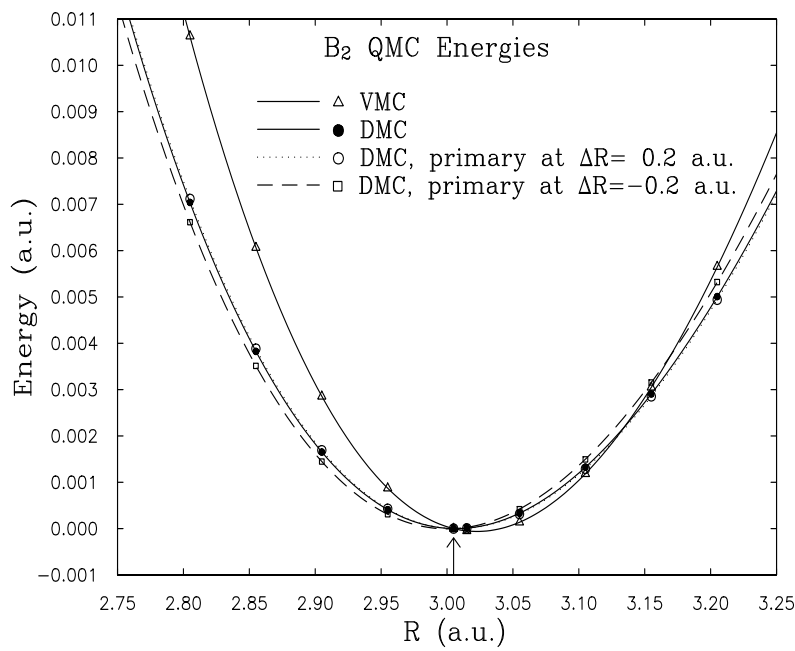

FIG. 2. Potential energy curve for $\mathrm{B}_{2}$ in VMC and DMC. The three DMC curves are obtained with three different primary geometries (equilibrium, stretched by 0.2 and -0.2 a.u.) and using recentered wave functions. All curves are shifted with the energy at the equilibrium distance (arrow) defined as the zero. Atomic units are used.

To test the improvement resulting from employing $\tau_{s} \neq \tau$ (Eq. 13), we performed, for $\mathrm{H}_{2}$, DMC correlated sampling with $\tau_{\mathrm{s}}=\tau$. Since $\tau_{\mathrm{s}}>\tau$ for $\Delta R>0$ and $\tau_{\mathrm{s}}<\tau$ for $\Delta R<0$, we expect this potential energy curve to yield an equilibrium bond-length that is too short. The equilibrium bond length is indeed 1.4003(2) a.u., which is 4 standard deviations from the true bond-length, whereas that obtained with our $\tau_{s} \neq \tau$ algorithm, 1.4014(2) a.u., is 1.5 standard deviation from the true bond-length.

Having ascertained the accuracy and efficiency of our algorithm, we computed the bond lengths of all first-row dimers with VMC and DMC correlated sampling. In Table 1 , we list the errors in the bond lengths obtained from restricted Hartree-Fock (RHF) [13], LDA [14], GGA [15], VMC and DMC. The RHF results show the worst agreement with experiment, with $\mathrm{Be}_{2}$ not being bound. The
DMC errors are, in all cases, either smaller than or comparable to those from VMC, and are smaller than LDA and GGA errors by a factor of 3.9 and 2.6, respectively.

In this letter, we presented an efficient method to compute numerical forces in DMC, a long-standing unsolved problem in QMC techniques. The method is very accurate and was tested on first-row dimers, where the DMC bond lengths were found to agree with experiment better than those from HF, LDA, GGA and VMC.

TABLE I. Experimental 16 bond lengths (in a.u.) of first-row dimers and theoretical errors in RHF, LDA, GGA, VMC and DMC.

\begin{tabular}{lcccccc}
\hline \hline molecule & Expt. & RHF & LDA & GGA & VMC & DMC \\
\hline $\mathrm{Li}_{2}$ & 5.051 & 0.270 & 0.069 & 0.057 & $0.101(2)$ & $0.018(3)$ \\
$\mathrm{Be}_{2}$ & 4.630 & - & -0.109 & -0.001 & $-0.069(3)$ & $-0.014(5)$ \\
$\mathrm{B}_{2}$ & 3.005 & 0.086 & 0.025 & 0.042 & $0.018(2)$ & $0.002(2)$ \\
$\mathrm{C}_{2}$ & 2.348 & -0.007 & 0.006 & 0.023 & $0.006(2)$ & $0.008(1)$ \\
$\mathrm{N}_{2}$ & 2.074 & -0.061 & -0.006 & 0.011 & $0.012(2)$ & $0.007(1)$ \\
$\mathrm{O}_{2}$ & 2.282 & -0.107 & -0.012 & 0.044 & $0.028(2)$ & $0.023(4)$ \\
$\mathrm{F}_{2}$ & 2.668 & -0.161 & -0.053 & 0.040 & $0.021(4)$ & $0.015(5)$ \\
$\mathrm{rms}$ & - & $\infty$ & 0.054 & 0.036 & 0.049 & 0.014 \\
\hline \hline
\end{tabular}

Acknowledgements. We thank S. Fahy for useful discussions. This work was begun during a visit to the Institute for Nuclear Theory in Seattle and is funded by Sandia National Laboratory.

[1] D. M. Ceperley and B. J. Alder, Phys. Rev. B 36, 2092 (1987); S. Fahy, X. W. Wang, and S. G. Louie, Phys. Rev. B 42, 3503 (1990); J. C. Grossman and L. Mitas, 79, 4353 (1997); R. Q. Hood et al., Phys. Rev. Lett. 78, 3350 (1997).

[2] C. J. Umrigar, K. G. Wilson, and J. W. Wilkins, Phys. Rev. Lett. 60, 1719 (1988).

[3] C. Filippi and C. J. Umrigar, J. Chem. Phys. 105, 213 (1996); for $\mathrm{O}_{2}$, we used an improved wave function.

[4] H. Hellman, Einführung in die Quanten Theorie (Deuticke, Leipzig, 1937); R. P. Feynman, Phys. Rev. 56, 340 (1939).

[5] K. C. Huang, R. J. Needs and G. Rajagopal, to be published.

[6] M. H. Kalos and P. A. Whitlock, Monte Carlo Methods, Vol. 1 (Wiley-Interscience 1986).

[7] R. E. Lowther and R. L. Coldwell, Phys. Rev. A 22, 14 (1980).

[8] C. J. Umrigar, Int. J. Quant. Chem. Symp. 23, 217 (1989).

[9] a) P. J. Reynolds et al., Int. J. Quan. Chem. 29, 589 (1986); b) C. A. Traynor and J. B. Anderson, Chem. Phys. Lett. 147, 389 (1988); c) J. Vrbik and S. M. Rothstein, J. Chem. Phys. 96, 2071 (1992).

[10] J.B. Anderson, J. Chem. Phys. 63, 1499 (1975); ibid. 65, 4121 (1976); P.J. Reynolds et al., ibid. 77, 5593 (1982).

[11] C. J. Umrigar, Nightingale and K.J. Runge, J. Chem. Phys. 99, 2865 (1993). 
[12] L. Wolniewicz, J. Chem. Phys. 99, 1851 (1993).

[13] K. A. Peterson, R. A. Kendall and T. H. Dunning, J. Chem. Phys. 99, 9790 (1993).

[14] A. D. Becke, Phys. Rev. A 33, 2786 (1986); R. M. Dickson and A. D. Becke, J. Chem. Phys. 99, 3898 (1993).

[15] F. W. Kutzler and G. S. Painter, Phys. Rev. B 45, 3236
(1992); they employ Perdew-Wang 86 GGA.

[16] K. P. Huber and G. Herzberg, Molecular Spectra and Molecular Structure. IV. Constants of Diatomic Molecules (Van Nostrand, Princeton, 1979); for $\mathrm{Be}_{2}, \mathrm{~V}$. E. Bondybey and J. H. English, J. Chem. Phys. 80, 568 (1984). 